\section{Composition and Surface Charge of DNA-Loaded Microparticles Determine Maturation and Cytokine Secretion in Human Dendritic Cells}

\author{
Samantha Jilek, ${ }^{1}$ Michael Ulrich, ${ }^{1}$ Hans P. Merkle, ${ }^{1}$ \\ and Elke Walter ${ }^{1,2}$
}

Received September 26, 2003; accepted February 1, 2004

\begin{abstract}
Purpose. Biodegradable microparticles prepared from poly(lactide) (PLA) and poly(lactide-co-glycolide) (PLGA) have been shown to be promising carrier systems for vaccine delivery. Here, we have investigated the capacity of different PLA and PLGA microparticle formulations to induce stimulation of human blood monocyte-derived dendritic cells (DCs).

Methods. Stimulation of human derived dendritic cells by plain microparticles were compared with microparticles loaded with plasmid DNA or double-stranded salmon DNA either by encapsulation or adsorption to the surface of cationic microparticles. Stimulation of DCs was monitored by the up-regulation of surface maturation markers CD83 and CD86 and the secretion of IL-12 and TNF- $\alpha$.

Results. Slowly degrading PLA microparticles did not induce any detectable stimulation or activation of DCs. In contrast, fast degrading PLGA microparticles were able to influence DC maturation and cytokine secretion dependent on their surface charge. Anionic PLGA microparticles induced an up-regulation of CD83 and high TNF- $\alpha$ secretion, which was further enhanced up to the level of the potent stimulator lipopolysaccharide (LPS) when plasmid DNA was encapsulated. Moreover, the secretion of significant amounts of IL-12 was observed. Cationic PLGA microparticles induced an up-regulation of CD86 and moderate TNF- $\alpha$ secretion, but no IL-12 secretion, with no additional effects in the presence of plasmid DNA.

Conclusions. The data suggest that the composition and charge of biodegradable DNA-loaded microparticles profoundly influences maturation and cytokine secretion in DCs. Thus, the individual formulation of microparticles used as a vaccine carrier system might considerably influence the profile of the immune response.
\end{abstract}

KEY WORDS: antigen-presenting cells; IL-12; poly(lactide-coglycolide) (PLGA); TNF- $\alpha$.

\section{INTRODUCTION}

Dendritic cells (DCs) play a key role in the induction of acquired and innate immunity (1). They are professional antigen presenting cells (APC) and are able to capture antigens, whole microbes, viruses, apoptotic bodies, and to a certain extent biodegradable microparticles $(2,3)$. Upon stimulation with inflammatory cytokines, lipopolysaccharide (LPS), or bacterial DNA $(1,4)$, DCs undergo a transition from the

\footnotetext{
${ }^{1}$ Department of Applied Biosciences, Swiss Federal Institute of Technology Zurich (ETH), 8057 Zurich, Switzerland.

${ }^{2}$ To whom correspondence should be addresed. (e-mail: elke_walter@ gmx.net)

ABBREVIATIONS: ad, addition; APC, antigen presenting cells; co, adsorption; DCs, dendritic cells; en, encapsulation; LPS, lipopolysaccharide; PEI, polyethylenimine; PLA, poly(D,L-lactide); PIDNA, double-straded plasmid DNA; PLGA, poly(D,L-lactide-co-glycolide); SaDNA, double-stranded salmon DNA.
}

immature to the mature state. Surface markers such as CD80, $\mathrm{CD} 83$, and CD86 become up-regulated, and secretion of immunostimulatory cytokines such as IL-12 and TNF- $\alpha$ is induced $(1,5,6)$. Mature DCs are able to induce an immune response by activating $\mathrm{T}$ cells in the secondary lymphoid organs (7). DCs also play a major role in determining the nature of the immune response, which may lead to tolerance or to $\mathrm{T}$ helper cell type 1 (Th1) or T helper cell type 2 (Th2) type immunity $(7,8)$. It has been suggested that the nature of immune response is directly correlated with the cytokine pattern that is produced by the DCs in response to different stimulatory signals (8). DCs have been shown to sense diverse pathogens and to subsequently elicit a tailored pathogenspecific immune response (9).

Biodegradable microparticles prepared from poly(lactide) (PLA) and poly(lactide-co-glycolide) (PLGA) have been shown to be potent antigen delivery systems for the induction of immune responses (10-12). Microparticles are valuable carriers for the targeting of antigens to APC because they have been demonstrated to be efficiently phagocytosed by DC in vitro $(3,13)$ and in vivo (14). In addition, phagocytosed microparticles release encapsulated antigen into the cells where it is efficiently presented on major histocompatibility complex (MHC) I and MHC II (15). Moreover, phagocytosed PLGA and PLA microparticles have been found to activate monocytes in vitro (16). Recently, uptake of PLGA nanoparticles has also been demonstrated to induce stimulation of DCs (17).

During recent years, DNA vaccination has been demonstrated to be efficient in the induction of immune responses (18) or protection from allergens (19). Moreover, bacterial DNA has direct immunostimulatory effects on DCs and represents a promising new class of vaccine adjuvants (4). Naked DNA is rapidly degraded in vivo (20). Therefore, the encapsulation of DNA in biodegradable microparticles is a particularly interesting approach that not only targets the APC but also enhances the amount of functional DNA delivered to these cells $(3,21,22)$.

In this study, we investigated the effect of various PLA and PLGA microparticles formulations on the stimulation of human monocyte-derived DCs. We compared plain microparticles with microparticles loaded with DNA either by encapsulation or adsorption to the microparticle surface. The various DNA-loaded microparticle formulations were analyzed for their in vitro release of DNA over time. Stimulation of DCs was assessed by the expression of specific surface markers and the secretion of IL-12 and TNF- $\alpha$. Our results demonstrate that synthetic biodegradable microparticles stimulate the maturation and the secretion of immunostimulatory cytokines in human DCs.

\section{MATERIALS AND METHODS}

\section{Materials}

Poly(D,L-lactide) (PLA; Resomer RG202H, MW 14,000) and poly(D,L-lactide-co-glycolide) (PLGA; Resomer RG502H, MW 13,700) were purchased from Boehringer Ingelheim (Ingelheim, Germany). Polyethylenimine (PEI), MW 600,000-1,000,000, was purchased from Fluka (Buchs, Switzerland). Crude double-stranded DNA from salmon testes 
was obtained from Sigma (Buchs, Switzerland). Green fluorescent protein (GFP) reporter gene plasmid was generated by cloning the GFP gene into the VR1012 vector. Plasmid was prepared with the Qiagen Giga Kit (Basel, Switzerland) according to the manufacturer's instructions and dissolved in nanopure water. Material for cell culture was purchased from Life Technologies AG (Basel, Switzerland). Lipopolysaccharide was obtained from Sigma (LPS; Escherichia coli 055:B5). All other chemicals used were of analytical grade unless otherwise specified and obtained by Fluka.

\section{Preparation and Characterization of Microparticles}

Various biodegradable PLA and PLGA microparticles were prepared by a spray-drying method described elsewhere $(21,23)$ (Table I). Briefly, for DNA encapsulation, DNA was dissolved in $0.1 \mathrm{M} \mathrm{NaHCO}_{3}$ and the resulting solution was dispersed in a $5 \%(\mathrm{w} / \mathrm{w})$ PLGA solution in ethyl formate by means of an ultrasonic processor (Vibra-Cell, Sonics \& Material, Danbury CT, USA). The w/o dispersions were spraydried in a laboratory spray dryer (Model 190, Büchi, Flawil, Switzerland). Microparticles were subsequently washed with a $0.1 \%(w / w)$ Pluronic F68 solution and water and dried under vacuum for $24 \mathrm{~h}$. Loading efficiency was determined as previously described (3). Cationic microparticles were prepared as described elsewhere (24). Briefly, PEI was dissolved in $0.2 \mathrm{M}$ phosphate buffer, $\mathrm{pH} 7.4$, which was subsequently dispersed in $10 \%(\mathrm{w} / \mathrm{w})$ PLGA or PLA solution in methylene chloride by means of an ultrasonic processor. The w/o dispersion was then spray-dried as stated above. Size distribution of the microparticle formulations was measured by laser light scattering (Mastersizer X, Malvern Instruments Ltd., Worcestershire, UK) and particle size was calculated based on Mie's theory (23). Zeta potential measurements were carried out in phosphate buffer at $\mathrm{pH} 7.4$ (ionic strength $=0.01$ ) by using a Zeta-Meter system 3 + (ZM3 + 331, Zeta-Meter, Inc., Staunton, VA, USA) $(3,24)$. DNA was loaded onto cationic microparticles prior to addition to the cells. One milligram of cationic microparticles was suspended in PBS, and $40 \mu \mathrm{g}$ of DNA was added and subsequently incubated for $4 \mathrm{~h}$ at $4{ }^{\circ} \mathrm{C}$ under gentle rotation. The DNA-loaded microparticles were then washed twice with PBS and resuspended in RPMI 1640 for further experiments. The microparticle preparations were tested for the presence of endotoxin using the QCL-1000 Chromogenic Limulus Amebocyte Lysate (LAL) kit (Biowhittaker, Verviers, Belgium). All microparticles were found to contain less than $5 \mathrm{EU} / \mathrm{mg}$ of endotoxin.

\section{Release of DNA from Microparticles}

DNA release from the different microparticle formulations was determined in PBS pH 5.5 at a microparticles concentration of $5 \mathrm{mg} / \mathrm{ml}$. The vials were incubated at $37^{\circ} \mathrm{C}$ under gentle shaking and samples were withdrawn after centrifugation and replaced by fresh PBS at regular time intervals. The amount of double-stranded DNA was assessed using the PicoGreen assay (Molecular Probes, Lucerne, Switzerland). Analysis was performed in 96-well plates according to the manufacturer's instructions using an automated plate reader (FluoroCount, Canberra Packard SA, Zurich, Switzerland) equipped with a filter set $485 / 530$.

Table I. Various Microparticle Formulations Tested for Their Effect on the Maturation of DCs

\begin{tabular}{llccc}
\hline \multicolumn{1}{c}{ Label* } & Polymer(s) $\dagger$ & Type DNA & $\begin{array}{c}\text { Association of DNA } \\
\text { with microparticles } \neq\end{array}$ & $\begin{array}{c}\text { Loading } \\
(\mu \mathrm{g} / \mathrm{mg})\end{array}$ \\
\hline PLA & PLA & - & - & 0 \\
PLA/SaDNA (ad) & PLA & Salmon & Addition & 40 \\
PLA/PIDNA (ad) & PLA & Plasmid & Addition & 40 \\
PLA/PEI & PLA, PEI & - & - & 0 \\
PLA/PEI/SaDNA (co) & PLA, PEI & Salmon & Adsorption & 30 \\
PLA/PEI/PIDNA (co) & PLA, PEI & Plasmid & Adsorption & 30 \\
\hline PLGA & PLGA & - & - & 0 \\
PLGA/SaDNA (en) & PLGA & Salmon & Encapsulation & 2.5 \\
PLGA/PIDNA (en) & PLGA & Plasmid & Encapsulation & 2.5 \\
PLGA/SaDNA (ad) & PLGA & Salmon & Addition & 40 \\
PLGA/PIDNA (ad) & PLGA & Plasmid & Addition & 40 \\
PLGA/PEI & PLGA, PEI & - & Adsorption & 30 \\
PLGA/PEI/SaDNA (co) & PLGA, PEI & Salmon & Adson & 30 \\
PLGA/PEI/PIDNA (co) & PLGA, PEI & Plasmid & Adsorption & \\
\hline
\end{tabular}

* PLA, poly(lactide); PLGA, poly(lactide-co-glycolide); PEI, polyethylenimine; PIDNA, plasmid DNA; SaDNA, double-stranded salmon DNA; co, adsorption; ad, addition; en, encapsulation.

$\dagger$ Microparticles containing PEI generally displayed a positive surface charge, which was maintained upon DNA adsorption, whereas all other microparticles displayed a negative surface charge. Determination was performed by zeta potential measurement and published in detail in a previous study (24).

$\$$ The DNA was associated to the various microparticles in three different ways: i) encapsulated during microparticle preparation (encapsulation); ii) adsorbed onto cationic microparticle surface (adsorption); iii) added to the dispersed microparticles in incubation medium (addition). 


\section{Cell Cultures}

DCs were obtained from human peripheral blood according to Sallusto et al. (25). Briefly, peripheral blood monocytes obtained from buffy coats (Blood-bank Zurich, Switzerland) were isolated by density gradient centrifugation on Ficoll-Paque (Pharmacia Biotech, Dubendorf, Switzerland). Peripheral blood monocytes were resuspended in RPMI 1640 supplemented with $10 \%$ heat inactivated (pooled) human serum (Blood-bank Zurich). The cells were then either frozen in freezing medium (10\% DMSO, 40\% FCS, 50\% RPMI 1640) and kept in liquid nitrogen for further analysis or used directly for the adherence in culture flasks $\left(25 \mathrm{~cm}^{2}\right)$ for $2 \mathrm{~h}$. Nonadherent cells were then removed, and the adherent cells were cultured in RPMI 1640 supplemented with 5\% heat inactivated (pooled) human serum in the presence of $1000 \mathrm{IU} /$ $\mathrm{ml} \mathrm{IL-4} \mathrm{(Sigma)} \mathrm{and} 50 \mathrm{ng} / \mathrm{ml}$ GM-CSF (R\&D Systems, Oxon, UK). Cultures were kept at $37^{\circ} \mathrm{C}$ in $5 \% \mathrm{CO}_{2}$ humidified atmosphere.

\section{Characterization of DCs}

Surface antigen expression of DCs was analyzed by flow cytometry. DCs were recovered from the flasks and washed once with plain medium. The DC were then incubated (45 min, $4^{\circ} \mathrm{C}$ ) with the following primary anti-human antibodies: CD11b/Mac-1 (Clone ICRF44, Pharmingen, San Diego, CA, USA), CD14 (Clone UCM-1, Sigma), CD83 (Clone HB15e, Pharmingen), and CD86 (Clone IT2.2, Pharmingen). Control cells were processed similarly with mouse isotype control antibodies IgG1 (MOPC-21, Sigma) and IgG2a (UPC-10, Sigma). Cells were washed once and subsequently incubated with the secondary antibody (anti-mouse IgG R-Phycoerythrin conjugate, Sigma) for $45 \mathrm{~min}$ at $4{ }^{\circ} \mathrm{C}$, washed twice and transferred to FACS tubes (Falcon, Becton Dickinson AG, Basel, Switzerland) for analysis by flow cytometry (FACScan, Becton Dickinson, San Jose, CA, USA). The cells were gated on the basis of their light-scattering properties. The results presented were corrected for nonspecific fluorescence, which was obtained from IgG isotype antibody control. Thus according to Santin and colleagues, more than $95 \%$ of the gated cells were identified as DCs (26).

\section{Assessment of DC Stimulation by Analysis of CD83 and CD86 Surface Expression}

At day 7 of culture, $10^{6}$ DCs were incubated with $300 \mu \mathrm{g}$ of the different microparticle formulations to investigate the influence of microparticle uptake on the maturation of DCs. DNA-adsorbed microparticles and microparticles with encapsulated DNA were directly added to cells. When incubated with PLA and PLGA microparticles, DC dispersions show substantial phagocytic uptake of the microparticles. Full experimental protocols were previously established by our group (3) and published together with associated data on phagocytosis. Controls were performed without any additions, or by adding the maturation inducer lipopolysaccharide ( $1 \mu \mathrm{g} / \mathrm{ml}$ ), or by adding $12 \mu \mathrm{g}$ of DNA together with the dispersed microparticles. After 72 h, DCs were analyzed for CD83 and CD86 surface expression by flow cytometry as described above. The results for CD83 surface marker for each donor were expressed as percent relative to LPS-stimulated DCs in order to eliminate the variations of the different do- nors (6). Accordingly, the results for CD86 surface marker were expressed as mean fluorescence intensity in relation to LPS-stimulated DCs.

\section{Analysis of Cytokine Secretion}

DCs $\left(10^{6}\right)$ were seeded in 6-wells, and microparticles or LPS were added as described above. After $24 \mathrm{~h}$, the supernatants were harvested and tested for the presence of IL12(p40), IL-12(p70) and TNF- $\alpha$ using an enzyme linkedimmunosorbent assay (ELISA; Pharmingen and Biosource International, Lucerna Chem, Luzern, Switzerland, respectively).

\section{Statistical Analysis}

Statistical analysis was performed using the nonpaired Student $t$ test. Nonstimulated DCs were compared to stimulated DCs. Values of $\mathrm{p}<0.05$ were considered to be statistically significant.

\section{RESULTS}

\section{Characterization of the Various DNA-Containing Microparticle Formulations}

Various types of biodegradable microparticles in the size range $1-10 \mu \mathrm{m}$ were prepared by using either PLA or PLGA polymer (Table II). Microparticles displaying a cationic surface charge were obtained by incorporation of the cationic polymer PEI (Table II). Phagocytosis by DCs has been investigated in detail in previous studies and revealed efficient uptake of all microparticle formlations $(3,24,27)$. In average, approximately 20 particles/cell were added in these experiments. Thus, based on previous results, phagocytosis is estimated to be about 10 particles/cell for anionic PLA or PLGA microparticles and about 20 particles/cell for cationic microparticles $(3,13,27)$. Anionic and cationic microparticles were loaded with two different types of DNA (Table I). Doublestranded salmon DNA or bacterial-derived plasmid DNA were encapsulated, resulting in a loading of about $2.5 \mu \mathrm{g}$ per mg microparticles, or adsorbed to the microparticle surface, with a loading of $30 \mu \mathrm{g}$ per mg microparticles (about 12-fold higher loading compared to encapsulated DNA) (Table I). In further control experiments, DNA was simply added to the

Table II. Particle Size and Zeta Potential of Anionic and Cationic PLGA and PLA Microparticles

\begin{tabular}{ccc}
\hline Microparticle type & Particle size $(\mu \mathrm{m})^{*}$ & Zeta potential $\dagger$ \\
\hline PLA & 5.6 & $-23.7(11.5)$ \\
PLA/PEI & 7.0 & $+36.9(3.2)$ \\
PLGA & 4.2 & $-65.9(11.2)$ \\
PLGA/PEI & 7.8 & $+11.0(0.5)$ \\
\hline
\end{tabular}

PLA, poly(lactide); PLGA, poly(lactide-co-glycolide); PEI, polyethylenimine.

* Calculation of particle sizes was based on Mie's theory accounting for the optical properties of the polymer, and particle size was expressed by using the average volume diameter [D4,3] in micrometers $(\mu \mathrm{m})$.

$\dagger$ Data are expressed as mean from $n=2$ and range is given in parenthesis. Loading with DNA did not significantly change the zeta potential. 
microparticles dispersed in the incubation medium at concentration of $40 \mu \mathrm{g}$ per mg microparticles.

In order to check to what extent DNA was released from the different microparticle formulations after phagocytosis, the microparticles were incubated in PBS pH 5.5, which corresponds to the $\mathrm{pH}$ present in phagolysosomes. The amount of DNA released over time was measured by analyzing the amount of double-stranded DNA (Fig. 1). As expected for PLGA microparticles containing encapsulated DNA $(3,21)$ a burst release of DNA took place during the first $24 \mathrm{~h}$ and is followed by a sustained release over at least 4 days (Fig. 1). In contrast, DNA encapsulated in PLA microparticles (up to 8 $\mu \mathrm{g} / \mathrm{mg}$ ) was only released in the initial burst phase and no sustained release could be detected (3). Based on this previous result, further studies including DNA encapsulated in PLA microparticles were not considered. When the DNA was simply added to the dispersed anionic PLA and PLGA microparticles, the whole quantity was detected in the incubation medium and no DNA was found to be bound to the microparticle surface (data not shown). In contrast, DNA was quantitatively adsorbed to cationic PLA and PLGA microparticles with no detectable release of DNA over 4 days, independently of the polymer type used (data not shown).

\section{Plain Microparticles Induce DC Maturation Depending on Polymer Type and Surface Charge}

We tested the influence of plain PLA and PLGA microparticles on DC maturation by measuring surface expression of CD83 and CD86. CD83 is a relevant marker for DC maturation and is exclusively expressed on mature DC (28), whereas CD86 is present on immature and mature DCs and is overexpressed upon DC stimulation (1). The various microparticle formulations were added to DC for 3 days and the cells were subsequently analyzed by flow cytometry for CD83 and CD86 surface expression. As controls, non-treated DCs and LPS-stimulated DCs were used. We found that none of the PLA microparticle formulations induced a significant upregulation of CD83 or CD86 expression (Fig. 2). In contrast, PLGA microparticles were able to influence DC maturation dependent on their surface charge. PLGA microparticles displaying a negative surface charge (Table I) induced upregulation of CD83. However they did not affect the expression of CD86 (Fig. 3). In contrast, CD86 was significantly up-regulated without affecting CD83 expression upon the ad-

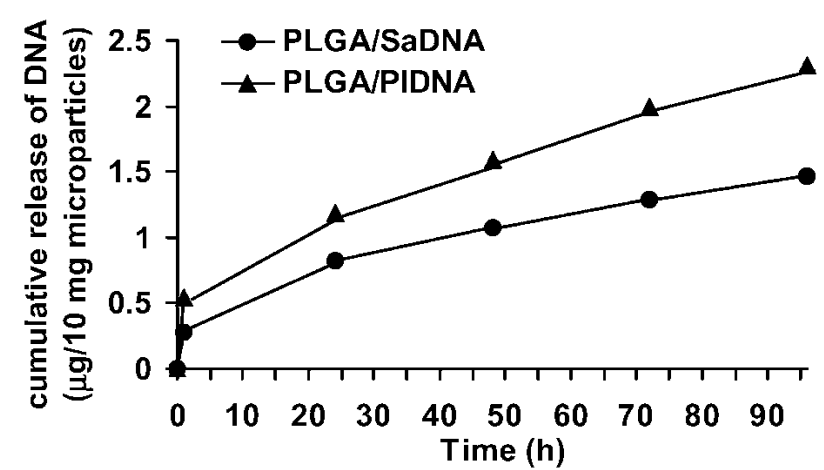

Fig. 1. Cumulative release over time of encapsulated doublestranded plasmid DNA (PIDNA) and salmon DNA (SaDNA) from PLGA microparticles. The data shown represent a typical set of two independent experiments for each microparticle formulation.
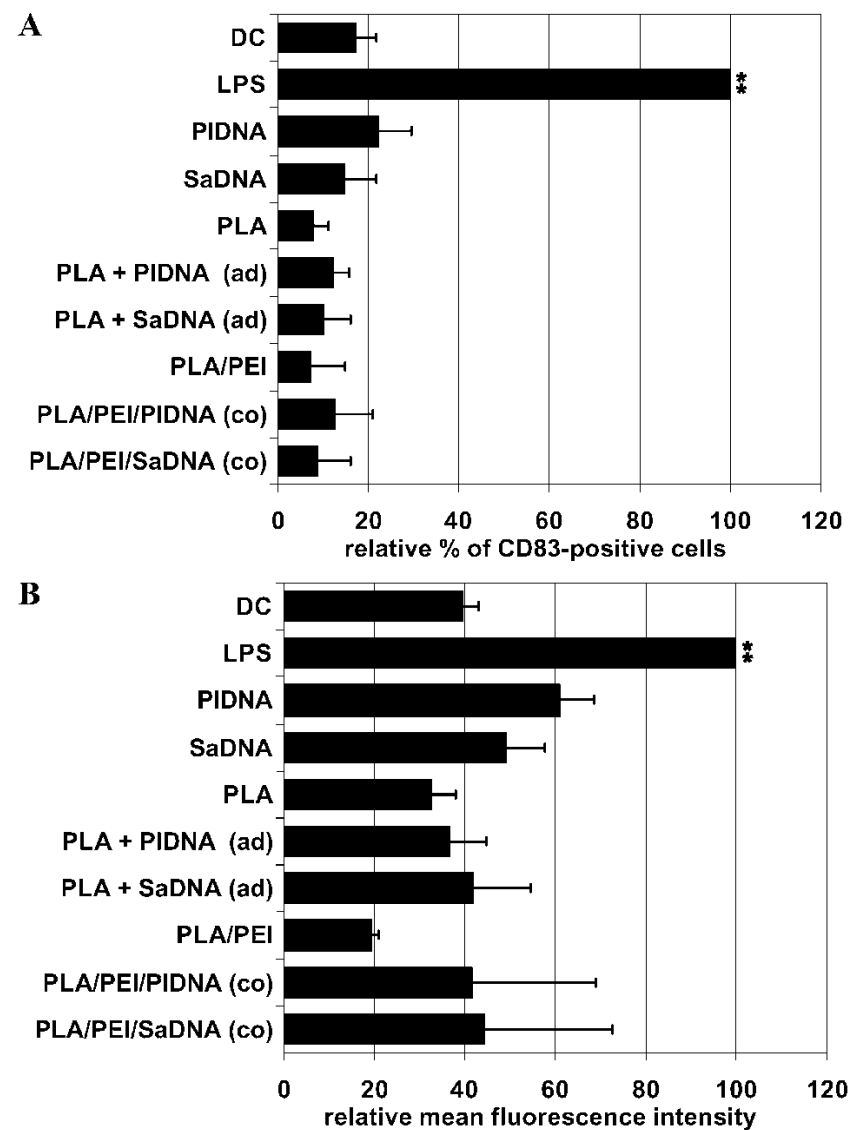

Fig. 2. Expression of (a) the maturation marker CD83 and (b) the activation marker CD86 upon addition of various PLA microparticle formulations. DCs cultured in the presence or absence of LPS were used as controls. The results for CD83 and CD86 surface marker for each donor were expressed as percent or MFI (mean fluorescence intentsity) relative to LPS-stimulated DCs in order to eliminate the variations of the different donors. Data are expressed as mean of $n=$ 2 to 4 independent experiments. Error bars represent standard error of means (SEM) ( $\mathrm{n}=3$ to 4$)$ or range between two experimental data sets $(n=2)$. Statistical analysis was performed using the nonpaired Student $t$ test $(* \mathrm{p}<0.05 ; * * \mathrm{p}<0.005)$. PIDNA $=$ plasmid DNA; SaDNA = salmon DNA; co = adsorption; ad = addition; en $=$ encapsulation.

dition of positively charged PLGA microparticles (Table I; Fig. 3).

In order to eliminate any effect of LPS contamination on the stimulation of DCs, all buffers and materials were checked for the presence of endotoxins by employing the LAL assay. No detectable LPS was found on materials and in the incubation buffers. The presence of LPS on the microparticle surfaces was found to be 3000 times less than the amount of LPS needed for the stimulation of the DCs.

\section{DNA-Loading Enhances Microparticle-Induced Maturation of DCs}

Bacterial DNA (4) or CpG containing unmethylated oligonucleotides very efficiently stimulate DCs (29), which is suggested to be an essential contribution for the success of DNA vaccination approaches (18). Thus, we checked whether microparticles containing DNA were able to further enhance the stimulating effect observed with plain microparticles. We 
$\mathbf{A}$

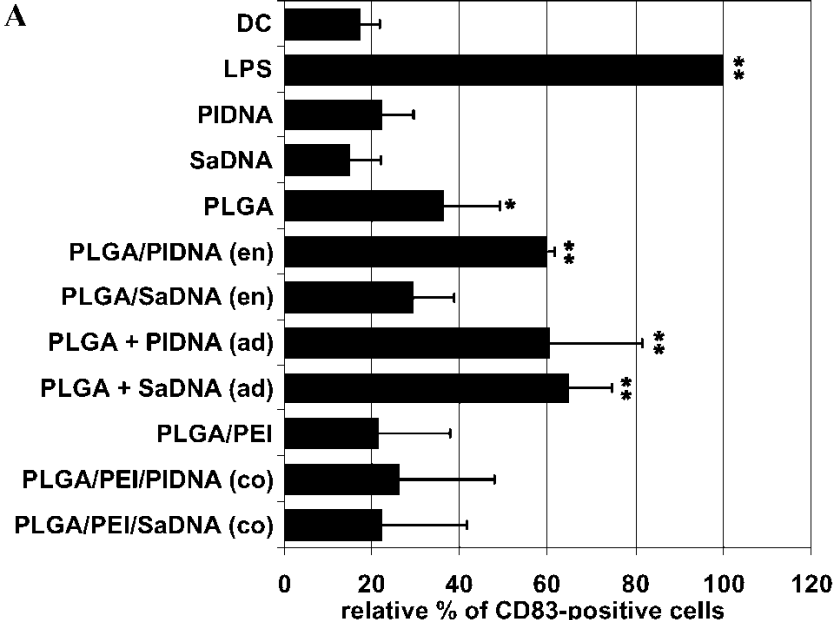

B

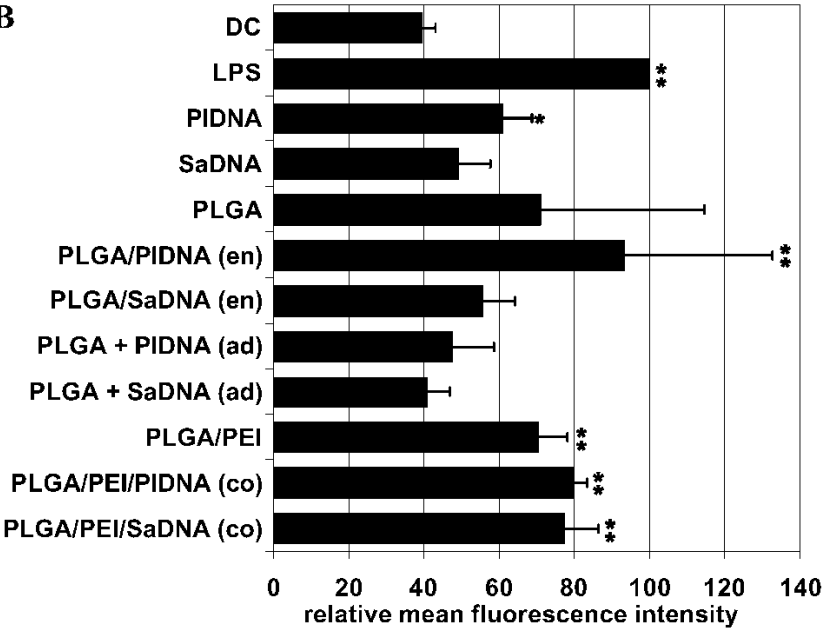

Fig. 3. Expression of (a) the maturation marker CD83 and (b) the activation marker CD86 upon addition of various PLGA microparticle formulations. DCs cultured in the presence or absence of LPS were used as controls. The results for CD83 and CD86 surface marker for each donor were expressed as percent or MFI relative to LPSstimulated DCs in order to eliminate the variations of the different donors. Data are expressed as mean of $n=2$ to 4 independent experiments. Error bars represent standard error of means (SEM) $(n=3$ to 4$)$ or range between two experimental data sets $(n=2)$. Statistical analysis was performed using the non-paired Student $t$ test $(* \mathrm{p}<0.05 ; * * \mathrm{p}<0.005)$. PIDNA = plasmid DNA; SaDNA $=$ double stranded salmon DNA; co = adsorption; ad = addition; en $=$ encapsulation.

used CpG-containing plasmid DNA and non-immunostimulatory salmon DNA as controls. Salmon DNA or plasmid DNA were either encapsulated or adsorbed to the microparticle surface. Control experiments were performed with soluble DNA or DNA added to the microparticle dispersion immediately before its addition to the cells (see Table I for details).

Even in the presence of adsorbed or added DNA, all microparticle formulations from PLA polymer were unable to induce up-regulation of surface CD83 or CD86 (Fig. 2). In contrast, PLGA microparticle-induced up-regulation of CD83 and CD86 was further enhanced when bacterialderived plasmid DNA was encapsulated in the negativelycharged PLGA microparticles (Fig. 3). A comparable amount of plasmid DNA alone was unable to induce up-regulation of CD83 and CD86 to the same extent. Similarly to results obtained with plain microparticles, positively charged DNAloaded PLGA microparticles induced an up-regulation of CD86 only which was not further enhanced upon administration of plasmid DNA. Furthermore, preliminary results indicated that a mixture of cationic and anionic PLGA microparticles induced a comparatively lower up-regulation of CD83 and CD86 (data not shown).

\section{PLGA Microparticles Stimulate the Secretion of IL-12 by DCs}

IL-12 is an important regulator of cell-mediated immune responses and is produced by mature and stimulated DC (5). The bioactive molecule IL-12(p70) is a heterodimer formed by two subunits (the p35 and the p40 subunit) (30). IL$12(\mathrm{p} 40)$ has been reported to be selectively produced by activated APC (31) and is secreted at a 100- to 1000-fold excess over the bioactive IL-12(p70) (30). Because PLGA microparticles were able to stimulate the maturation of $\mathrm{DC}$, we checked whether in addition, the secretion of the bioactive IL-12(p70) or of IL-12(p40) was induced. Various PLGA microparticle formulations were added to DCs for $24 \mathrm{~h}$, and the culture medium was tested for secreted IL-12(p70) and IL12(p40), respectively, by ELISA. No secretion of IL-12(p70) above detection limit of the assay was found for any of the polymer formulations tested (same formulations as in Fig. 4). Control experiments with LPS-activated DCs revealed moderate secretion of IL-12(p70) (ranging from $50 \mathrm{pg} / \mathrm{ml}$ up to $550 \mathrm{pg} / \mathrm{ml}$ ) indicating the functionality of the assay. Plain PLA microparticles were not able to induce significant production of IL-12, therefore other PLA formulations were not further considered. On the other hand, IL-12(p40) was found to be secreted at high levels upon addition of already plain anionic PLGA microparticles. As the addition of soluble plasmid DNA induced noticeable secretion of IL-12(p40) (Fig. 4), its enhancing effect on IL-12 secretion was cumulative when ad-

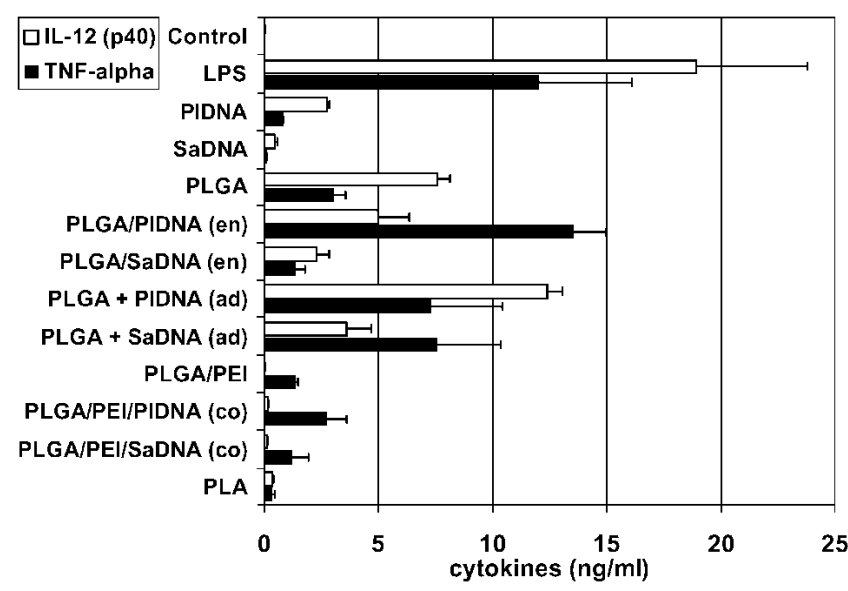

Fig. 4. Production of IL-12 and TNF- $\alpha$ after uptake of various PLGA and PLA microparticle formulations. DCs cultured in the presence or absence of LPS were used as controls. Data are expressed as mean of $\mathrm{n}=2$ to 4 independent experiments. Error bars represent standard error of means (SEM) $(\mathrm{n}=3=$ to 4$)$ or range between two experimental data sets $(n=2)$. PIDNA = plasmid DNA; SaDNA = double stranded salmon DNA; co = adsorption; ad = addition; en = encapsulation. 
ministered together with plain anionic PLGA microparticles, however not with DNA encapsulated in the microparticles. As expected, the presence of salmon DNA did not show any significant enhancement of IL-12 secretion. In contrast, anionic PLA microparticles and cationic PLGA microparticles did not induce any detectable secretion of IL-12(p40), even in the presence of plasmid DNA.

\section{PLGA Microparticles Stimulate the Secretion of TNF- $\alpha$ by DCs}

TNF- $\alpha$ is an inflammatory cytokine and an important regulator of the immune response. It is secreted by DCs at a very early stage of stimulation and has been suggested to play a role in the activation of DCs $(5,6)$, in Langerhans cell migration (32), and in the recruitment of DCs to the site of inflammation (6). In this study, we found that the secretion of TNF- $\alpha$ upon the addition of anionic PLA and PLGA microparticles correlated fairly well with the secretion of IL-12 (Fig. 4). PLGA microparticles induced a significant production of TNF- $\alpha$, whereas PLA did not. Moreover, TNF- $\alpha$ secretion was noticeably enhanced to the level of LPS-stimulated cells when plasmid DNA was encapsulated in PLGA microparticles. The induction of TNF- $\alpha$ secretion was generally in good accordance with DC maturation (CD83 up-regulation) (Figs. 3a and 3b). However, PLGA cationic microparticles induced a moderate TNF- $\alpha$ secretion, although we did not observe detectable DC maturation or IL-12(p40) production.

\section{DISCUSSION}

The search for efficient and safe synthetic vaccine carrier systems has yielded various types of biodegradable anionic and cationic microparticles, which are able to induce noticeable immune responses in vivo when loaded with peptide or protein antigens $(10,23,33)$ or plasmid DNA $(11,34)$. The adequate stimulation of DCs is of key importance for the initiation of acquired and innate immune responses by these cells. Thus, the aim of this study was to investigate the role of composition and charge of synthetic biodegradable microparticle formulations on the maturation and cytokine secretion of human DCs. We found that DCs exhibited microparticlespecific stimulation patterns, which greatly depended on the polymer formulation. To our knowledge, this is the first study in vitro demonstrating the induction of DC maturation and cytokine production upon exposure to PLGA microparticles, which are widely tested as biodegradable synthetic vaccine carrier systems.

CD83 and CD86 play important roles in the activation of T-cells and were shown to be highly up-regulated in DCs found in the lymph nodes (35). Both surface markers have been used to detect maturation of DCs. However, their role for the function of DCs in the course of the immune response must be clearly distinguished. CD83 is an adhesion receptor present only on the surface of mature DCs (28). It is believed that CD83 is able to bind to its counter-receptor expressed on monocytes and activated $\mathrm{T}$ cells, and thus, has an important role in the induction and regulation of the immune response (36). Both CD86 and CD80 are co-stimulatory molecules and participate in directing the immune response toward Th1 or Th2 response. In addition, interrupting the pathway of costimulation leads to the inhibition of T-cells and results in an antigen-specific hyporesponsiveness (37). Mature DCs can either be fully activated and express high surface levels of costimulatory molecules or are not yet fully activated and express moderate levels of co-stimulatory molecules (38). It is hypothesised that the level of activation of mature DCs is likely to determine the initiation of a protective immune response or of tolerance (38).

Already plain PLGA microparticles were able to significantly induce an up-regulation of the surface expression of either CD83 or CD86, which was strongly dependent on the surface charge of the microparticles. Whereas anionic PLGA microparticles induced the up-regulation of CD83 and CD86, cationic PLGA microparticles were able to up-regulate CD86 only. So far, we can not fully explain these findings, but it is suggested that the surface charge of the carrier may play an important role in inducing or even inhibiting the maturation of DCs.

Furthermore, we found that CpG-containing plasmid DNA encapsulated in PLGA microparticles strongly enhanced the up-regulation of both CD83 and CD86, which was already induced by plain microparticles. Salmon DNA, which is $\mathrm{CpG}$ deficient, was non-immunostimulatory. An upregulation of CD83, but not of CD86 was already seen in the case of simply adding soluble plasmid DNA to the microparticle dispersion. However, although the total amount of DNA administered in its soluble form was 12-fold higher, the effect on DCs stimulation was comparable (CD83 up-regulation) or even significantly higher (CD86-up-regulation) when DNA was encapsulated in the microparticles. These findings underline the importance of a physical connection between both stimulatory agents. Thus, we conclude that efficient uptake of DNA-loaded microparticles by DCs, as demonstrated in a previous study (3), is likely to enhance DC stimulation due to the intracellular delivery of comparably high amounts of DNA. This is because after microparticle uptake, the encapsulated DNA is released upon degradation of the PLGA microparticle matrix inside the cells (3).

Interestingly, adsorption of comparably high amounts of plasmid DNA to the surface of cationic PLGA microparticles did not enhance the up-regulation of CD83 or CD86 as compared to plain cationic PLGA microparticles. This could be explained by the fact that the DNA was not released from the surface of the cationic PLGA microparticles, as demonstrated in the in vitro release assays.

In contrast to the results obtained with PLGA microparticles, we found that none of the PLA microparticle formulations was able to induce detectable stimulation of DCs. There appears to be no major difference in the microparticles size and surface charge, the amount of DNA loading and the amount of microparticles phagocytosed per cell (3) between the PLA and PLGA microparticle formulations which could explain the difference in DC stimulation. Therefore, we hypothesize that the stimulatory effect of the PLGA microparticles may be due to the chemical composition of the polymer. Although both polymers are insoluble in water, PLGA microparticles are more hydrophilic than PLA microparticles. This leads to a rapid uptake of water into the microparticle core and subsequent swelling of the PLGA microparticles (39). The presence of water inside the microparticles enhances polymer hydrolysis and degradation leading to a relatively fast intracellular release of lactic and glycolic acid. Indeed, PLGA microparticles completely degrade inside DCs 
within 10 days (3). In contrast, PLA microparticles degrade very slowly over several weeks (23). These data imply that the material, composition and rate of degradation of the carrier itself may play an important role to induce stimulation of DCs. At this point it is unknown whether the more rapid release of acidic moieties from PLGA microparticles vs. PLA microparticles would fully explain the observed difference in DC stimulation. Nevertheless, we observed up-regulation of the expression of inflammatory marker genes in the presence of plain biodegrading PLGA microparticles in bone defects (V. Luginbühl, L. Meinel, unpublished data).

The immunostimulatory capacity of DCs not only depends on the up-regulation of membrane-bound signals, but also on the secretion of soluble signals, such as cytokines. Our results show that the secretion of two potent stimulators IL-12 and TNF- $\alpha$ was induced upon the addition of anionic PLGA microparticles, but not by PLA microparticles. These findings are in agreement with results obtained by others, showing that CD83 expression is a necessary factor, although not sufficient, to induce a high level of IL-12 in DC (40). Although we found considerable amounts of the IL-12 p40 subunit, the production of the bioactive IL-12 p40/p35 heterodimer was below the detection limit of our assay. This could be explained by the presence of TNF- $\alpha$ in the cell culture medium, which has been demonstrated to be secreted very early upon DC stimulation and to inhibit the induction of IL-12 p35 mRNA to barely detectable levels (6).

Stimulation of maturation as well as the production of the bioactive form of IL-12 in human DC has been previously reported upon addition of soluble DNA (4). However, only a specific plasmid DNA, containing two repeats of the strong immunostimulatory motifs $5^{\prime}$-AACGTT-3', was able to display these effects at concentrations as used in our study. Here, we used a plasmid DNA containing only weak immunostimulatory sequences (4) which could explain the absence of CD83 up-regulation and the weak up-regulation of CD86 in the presence of the plasmid alone. In this context it is noteworthy to mention that the mechanistic role of $\mathrm{CpG}$ on monocyte-derived DC is controversial (4,29).

Encapsulated plasmid DNA enhanced the TNF- $\alpha$ secretion up to the level of the potent stimulator LPS, which is in accordance with DC maturation. In contrast, encapsulated plasmid DNA did not further increase IL-12 production compared to plain PLGA microparticles, which is likely to be due to the early time point in IL-12 determination when DNA release from microparticles was not complete. Interestingly, moderate TNF- $\alpha$ secretion was also found with cationic PLGA microparticles which did not induce detectable IL-12 secretion or DC activation.

Thus, DCs exhibited stimulus-specific maturation and cytokine production rather than generating a universal core response to the various microparticle formulations. This effect is in agreement with findings that diverse pathogens elicit tailored pathogen-specific immune responses triggered by DCs (9). Moreover, our study indicates that a tailored stimulation of DC maturation and activation can also be induced by fully synthetic biodegradable microparticles. We hypothesise that in addition to inducing full DC stimulation, anionic PLGA microparticles containing encapsulated plasmid DNA may play a direct role in the development of IFN- $\gamma$-producing Th1 cells (41), which is important for cell-mediated immune responses.
In summary, we found that the character of the biodegradable microparticles profoundly influences the response of DCs in terms of maturation and cytokine production. Thus, we demonstrated a plasticity of DC responses to various fully synthetic microparticles that was previously only reported for pathogens or live vaccine vectors (9). Anionic PLGA microparticles in combination with encapsulated plasmid DNA appeared to be potent stimulators for the induction of the DC maturation markers CD83 and CD86. On the other hand, the combination of the PLGA polymer with a cationic surface led to the up-regulation of the surface marker CD86 only. Comparably high levels of both IL-12 and TNF- $\alpha$ were only produced upon addition of anionic PLGA microparticles, but not by cationic microparticles or PLA microparticles. We therefore postulate that the choice of the microparticles formulation used as a carrier system for DNA vaccines may strongly influence the outcome of the immune response. Especially, the anionic PLGA microparticles, which stimulated maturation of DCs as well as production of IL-12 and TNF- $\alpha$ appear promising for future vaccine applications.

\section{ACKNOWLEDGMENTS}

This work was supported by the Swiss National Research Foundation (grant no. 4037-55144). We thank Dr. Lars Thiele for his excellent help in culturing human DC and Dr. Donatus Dreher (Department of Pneumology, University Hospital of Geneva) for his help with flow cytometry and for critical reading, comments and suggestions.

\section{REFERENCES}

1. J. Banchereau, F. Briere, C. Caux, J. Davoust, S. Lebecque, Y.-J. Liu, B. Pulendran, and K. Palucka. Immunobiology of dendritic cells. Annu. Rev. Immunol. 18:767-811 (2000).

2. S. Greenberg and S. Grinstein. Phagocytosis and innate immunity. Curr. Opin. Immunol. 14:136-145 (2002).

3. E. Walter, D. Dreher, M. Kok, L. Thiele, S. G. Kiama, P. Gehr, and H. P. Merkle. Hydrophilic poly(DL-lactide-co-gylcolide) microspheres for the delivery of DNA to human-derived macrophages and dendritic cells. J. Control. Rel. 76:149-168 (2001).

4. D. Schattenberg, M. Schott, G. Reindl, T. Krueger, D. Tschoepe, J. Feldkamp, W. A. Scherbaum, and J. Seissler. Response of human monocyte-derived dendritic cells to immunostimulatory DNA. Eur. J. Immunol. 30:2824-2831 (2000).

5. B. de Saint-Vis, I. Fugier-Vivier, C. Massacrier, C. Gaillard, B. Vanbervliet, S. Ait-Yahia, J. Banchereau, Y.-J. Liu, S. Lebecque, and C. Caux. The cytokine profile expressed by human dendritic cells is dependent on cell subtype and mode of activation. J. Immunol. 160:1666-1676 (1998).

6. A. Langenkamp, M. Messi, A. Lanzavecchia, and F. Sallusto. Kinetics of dendritic cell activation: impact on priming of Th1, Th2 and nonpolarized T cells. Nat. Immunol. 1:311-316 (2000).

7. P. Guermonprez, J. Valladeau, L. Zitvogel, C. Théry, and S. Amigorena. Antigen presentation and $\mathrm{T}$ cell stimulation by dendritic cells. Annu. Rev. Immunol. 20:621-667 (2002).

8. Y. Wanand and J. Bramson. Role of dendritic cells-derived cytokines in immune regulation. Curr. Pharm. Des. 7:977-992 (2001).

9. Q. Huang, D. Liu, P. Majewski, L. C. Schulte, J. M. Korn, R. A. Young, E. S. Lander, and N. Hacohen. The plasticity of dendritic cell responses to pathogens and their components. Science 294: 870-875 (2001).

10. Y. Men, H. Tamber, R. Audran, B. Gander, and G. Corradin. Induction of a cytotoxic $\mathrm{T}$ lymphocyte response by immunization with a malaria specific CTL peptide entrapped in biodegradable polymer microspheres. Vaccine 15:1405-1412 (1997).

11. M. L. Hedley, J. Curley, and R. Urban. Microspheres containing 
plasmid-encoded antigens elicit cytotoxic T-cell responses. Nat. Med. 4:365-368 (1998).

12. P. Johansen, Y. Men, H. P. Merkle, and B. Gander. Revisiting PLA/PLGA microspheres: an analysis of their potential in parenteral vaccination. Eur. J. Pharm. Biopharm. 50:129-146 (2000).

13. L. Thiele, B. Rothen-Rutishauser, S. Jilek, H. WunderliAllenspach, H. P. Merkle, and E. Walter. Evaluation of particle uptake in human blood monocyte-derived cells in vitro. Does phagocytosis activity of dendritic cells measure up with macrophages? J. Control. Rel. 76:59-71 (2001).

14. K. D. Newman, P. Elamanchili, and G. S. Kwon. and J. Samuel. Uptake of poly(D,L-lactic-co-glycolic acid) microspheres by antigen-presenting cells in vivo. J. Biomed. Mater. Res. 60:480-486 (2002).

15. Y. Men, R. Audran, C. Thomasin, G. Eberl, S. Demotz, H. P. Merkle, B. Gander, and G. Corradin. MHC class I- and class II-restricted processing and presentation of microencapsulated antigens. Vaccine 17:1047-1056 (1999).

16. S. Prior, B. Gander, N. Blarer, H. P. Merkle, M. L. Subira, J. M. Irache, and C. Gamazo. In vitro phagocytosis and monocytemacrophage activation with poly(lactide) and poly(lactide-coglycolide) microspheres. Eur. J. Pharm. Sci. 15:197-207 (2002).

17. M. E. Lutsiak, D. R. Robinson, C. Coester, and G. S. Kwon. and J. Samuel. Analysis of poly(D,L-lactic-co-glycolic acid) nanospheres uptake by human dendritic cells and macrophages in vitro. Pharm. Res. 19:1480-1487 (2002).

18. S. Gurunathan, D. M. Klinman, and R. A. Seder. DNA vaccines: Immunology, application, and optimization. Annu. Rev. Immunol. 18:927-974 (2000).

19. S. Jilek, C. Barbey, F. Spertini, and B. Corthesy. Antigenindependent suppression of the allergic immune response to bee venom phospholipase $\mathrm{A}(2)$ by DNA vaccination in $\mathrm{CBA} / \mathrm{J}$ mice. J. Immunol. 166:3612-3621 (2001).

20. D. Lew, S. E. Parker, T. Latimer, A. M. Abai, A. KuwaharaRundell, S. G. Doh, Z. Y. Yang, D. Laface, S. H. Gromkowski, and G. J. Nabel. and al. Cancer gene therapy using plasmid DNA: pharmacokinetic study of DNA following injection in mice. Hum. Gene Ther. 6:553-564 (1995).

21. E. Walter, K. Moelling, J. Pavlovic, and H. P. Merkle. Microencapsulation of DNA using poly(DL-lactide-co-glycolide): stability issues and release characteristics. J. Control. Rel. 61:361-374 (1999).

22. A. M. Tinsley-Bown, R. Fretwell, A. B. Dowsett, S. L. Davis, and G. H. Farrar. Formulation of poly(D,L-lactic-co-glycolic acid) microparticles for rapid plasmid DNA delivery. J. Control. Rel. 66:229-241 (2000).

23. C. Thomasin, G. Corradin, Y. Men, H. P. Merkle, and B. Gander. Tetanus toxoid and synthetic malaria antigen containing poly(lactide)/poly(lactide-co-glycolide) microspheres: importance of polymer degradation and antigen release for immune response. J. Control. Rel. 41:131-145 (1996).

24. E. Walter and H. P. Merkle. Microparticle-mediated transfection of non-phagocytic cells in vitro. J. Drug Target. 10:11-21 (2002).

25. F. Sallusto, M. Cella, C. Danieli, and A. Lanzavecchia. Dendritic cells use macropinocytosis and the mannose receptor to concentrate macromolecules in the major histocompatibility complex class II compartment: downregulation by cytokines and bacterial products. J. Exp. Med. 182:389-400 (1995).
26. A. D. Santin, P. L. Hermonat, A. Ravaggi, M. Chiriva-Internati, M. J. Cannon, J. C. Hiserodt, S. Pecorelli, and G. P. Parham. Expression of surface antigens during the differentiation of human dendritic cells vs macrophages from blood monocytes in vitro. Immunobiology 200:187-204 (1999).

27. S. Faraasen, J. Vörös, G. Csucs, M. Textor, H. P. Merkle, and E. Walter. Ligand-specific targeting of microspheres to phagocytes by surface modification with poly(L-lysine)-grafted poly(ethylene glycol) conjugate. Pharm. Res. 20:237-246 (2003).

28. L.-J. Zhou and T. F. Tedder. CD14+ blood monocytes can differentiate into functionally mature CD83+ dendritic cells. Proc. Natl. Acad. Sci. U.S.A. 93:2588-2592 (1996).

29. G. Hartmann, G. J. Weiner, and A. M. Krieg. CpG DNA: A potent signal for growth, activation, and maturation of human dendritic cells. Proc. Natl. Acad. Sci. U.S.A. 96:9305-9310 (1999).

30. G. Carra, F. Gerosa, and G. Trinchieri. Biosynthesis and posttranslational regulation of human IL-12. J. Immunol. 164:47524761 (2000).

31. F. Takeshita and D. M. Klinman. CpG ODN-mediated regulation of IL-12 p40 transcription. Eur. J. Immunol. 30:1967-1976 (2000).

32. B. Wang and D. N. Sauder. Role of cytokines in epidermal Langerhans cell migration. J. Leukoc. Biol. 66:33-39 (1999).

33. K. D. Newman, J. Samuel, and G. Kwon. Ovalbumin peptide encapsulated in poly(d,1 lactic-co-glycolic acid) microspheres is capable of inducing a T helper type 1 immune response. J. Control. Rel. 54:49-59 (1998).

34. K. S. Denis-Mize, M. Dupuis, M. L. MacKichan, M. Singh, B. Doe, and D. O'Hagan. J. B. UImer, J. J. Donnelly, D. M. McDonald, and G. Ott. Plasmid DNA adsorbed onto cationic microparticles mediates target gene expression and antigen presentation by dendritic cells. Gene Ther. 7:2105-2112 (2000).

35. K. Takahashi, K. Asagoe, J. Zaishun, H. Yanai, T. Yoshino, K. Hayashi, and T. Akagi. Heterogeneity of dendritic cells in human superficial lymph node: in vitro maturation of immature dendritic cells into mature or activated interdigitating reticulum cells. $\mathrm{Am}$. J. Pathol. 153:745-755 (1998).

36. N. Scholler, M. Hayden-Ledbetter, A. Dahlin, I. Hellström, K.-E. Hellström, and J. A. Ledbetter. Cutting Edge: CD83 regulates the development of cellular immunity. J. Immunol. 168:25992602 (2002).

37. D. J. Lenschow, T. L. Walunas, and J. A. Bluestone. CD28/B7 system of T cells costimulation. Annu. Rev. Immunol. 14:233-258 (1996).

38. K. Shortman and Y.-J. Liu. Mouse and human dendritic cell subtypes. Nat. Rev. Immunol. 2:151-161 (2002).

39. M. A. Tracy, K. L. Ward, L. Firouzabadian, Y. Wang, N. Dong, R. Qian, and Y. Zhang. Factors affecting the degradation rate of poly(lactide-co-glycolide) microspheres in vivo and in vitro. Biomaterials 20:1057-1062 (1999).

40. P. J. Mosca, A. C. Hobeika, T. M. Clay, S. K. Nair, E. K. Thomas, M. A. Morse, and H. K. Lyerly. A subset of human monocytederived dendritic cells expresses high levels of interleukin-12 in response to combined CD40 ligand and interferon- $\gamma$ treament. Blood 96:3499-3504 (2000).

41. M. Moser and K. M. Murphy. Dendritic cell regulation of Th1Th2 development. Nat. Immunol. 1:199-205 (2000). 